\title{
Childhood factors that predict asthma in young adulthood
}

\author{
B.G. Toelle*, W. Xuan*, J.K. Peat", G.B. Marks*
}

Childhood factors that predict asthma in young adulthood. B. G. Toelle, W. Xuan, J.K. Peat, G.B. Marks. C) ERS Journals Ltd 2004.

ABSTRACT: Predicting adult asthma, using childhood characteristics, is important for advising on prognosis and, potentially, for secondary prevention. A novel use of multivariate likelihood ratios (LRs) to quantify prognosis is described here.

Of 718 subjects of a community-based cohort, $575(80 \%)$ members were recruited at age 8-10 yrs and were re-assessed 15-17 yrs later. At baseline, information about symptoms, spirometry, histamine challenge and skin-prick tests were collected. At follow-up "asthma symptoms" were defined as wheeze, sleep disturbance from asthma or inhaled steroid use within the previous year. LRs were calculated for significant predictors of this outcome. Shinkage factors were applied to yield multivariate LRs.

Childhood characteristics that independently predicted asthma symptoms in adulthood were obstructive spirometry (adjusted (adj)LR 2.9, 95\% confidence interval (CI) 1.3-6.5), airway hyperresponsiveness (adjLR 2.6, 95\% CI 1.8-3.7), atopy (adjLR 2.0, 95\% CI 1.5-2.7), recent wheeze (adjLR 1.9, 95\% CI 1.5-2.5) and being female (adjLR 1.29, 95\% CI 0.8-2.1). Children with all five characteristics had a cumulative LR of 36.9 for asthma symptoms in adulthood.

Most adults who had asthma symptoms did not have manifestations of asthma as children. However, the presence of obstructive spirometry, airway hyperresponsiveness and atopy in childhood identifies individuals with increased likelihood of having asthma in adulthood. Cumulative likelihood ratios are more valuable than odds ratios for quantifying risk in individuals and for identifying people with most to gain from preventive interventions.

Eur Respir J 2004; 23: 66-70.

\begin{abstract}
*Woolcock Institute of Medical Research, and ${ }^{\#}$ Dept of Paediatrics and Child Health, University of Sydney, Sydney, Australia.
\end{abstract}

Correspondence: B.G. Toelle, Box M77 Missenden Road Post Office, Camperdown, New South Wales 2050, Australia.

Fax: 61295506115

E-mail: bgt@woolcock.org.au

Keywords: Asthma

prognosis

risk factors

Received: April 292003

Accepted after revision: September 32003

This work was supported by Allen+Hanburys (Respiratory Devision of GlaxoSmithKline, Boronia, Victoria), AstraZeneca (North Ryde, Sydney), National Health and Medical Research Council (Canberra), Asthma New South Wales (Sydney), and Community Health and Tuberculosis Australia (Sydney). The funding sources only contributed financially to this study.
There are several reasons for identifying childhood characteristics that predict the subsequent likelihood of having asthma. Parents often request simple prognostic information from doctors dealing with their allergic and wheezy children. Such information is valuable in targeting treatments and preventive interventions at a high-risk population group. Finally, identifying the childhood antecedents of adult asthma would improve the understanding of the mechanisms underlying this disease. This information can only be gained by following cohorts over a period that encompasses the transition from childhood to adult life. There have been relatively few such studies.

Several existing cohort studies, some based on general population cohorts of children and others based on cohorts of children with various forms of asthma, have been reported from Australia [1-3], New Zealand [4], the UK [5, 6] and the Netherlands [7]. Important conclusions from these studies are that children with asthma or asthma-like symptoms who have more frequent symptoms are more likely to have disease persisting into adult life [8], and that the presence, during childhood, of atopy, airway hyperresponsiveness (AHR) and airflow obstruction $[9,10]$, predicts either the persistence of childhood asthma symptoms or their onset in adult life. Furthermore, the presence of AHR during childhood is associated with the development of airflow obstruction during subsequent lung growth and development [9], and all of the above features, together with early onset of symptoms and low lung function, predict an increased risk of subsequent hospitalisation among children with a history of wheeze [11].
Existing forms of data presentation do not allow the application of these data to the quantitative assessment of prognosis in an individual child. Likelihood ratios (LRs) have been popularised as a tool to assist in clinical diagnosis by quanifying the extent to which a specific test result increases or decreases the prior odds that disease is present [12]. Several nonindependent clinical findings can be incorporated into a diagnostic algorithm by using a multivariate method to derive independent likelihood ratios [13, 14]. In the analysis presented here, using data from the Belmont cohort study, this method has been adapted to the assessment of prognosis.

The aim of this report is to present an algorithm for predicting, on the basis of childhood characteristics, whether an individual will manifest asthma symptoms in adult life. The predicting algorithm incorporates a range of factors and is applicable in children with, and without, diagnosed asthma. The algorithm may be used to quantitatively predict the prognosis for asthma outcomes in individual children.

\section{Methods}

\section{Subjects}

The subjects were members of a cohort of 718 children aged 8-10 yrs who were recruited in the winter months (June and July) of 1982 from a random selection of primary schools in the Belmont region of New South Wales [3]. The initial participation rate was $87 \%$ of eligible subjects. The cohort 
was followed up each second year until 1992 and then again in 1997-1999. During 1997-1999, 80\% of the tests were conducted during the winter months. All studies were approved by the Research Ethics Committee of the University of Sydney. Informed consent was obtained from all subjects or their parents prior to each study.

\section{Respiratory symptoms, family history, asthma management and morbidity}

In 1982, a questionnaire was completed by the subjects' parents. During 1997-1999 the subjects, now adults, completed the questionnaire themselves. At both studies, standardised questions asked whether the subject had ever had wheeze, wheeze during or after exercise, or night cough. They were asked whether they had ever used any medication for asthma or had ever had asthma diagnosed by a doctor. Those who had ever had symptoms were also asked if they had these symptoms in the preceding 12 months, i.e. recent symptoms. The initial questionnaire also collected data on family history of asthma, eczema and rhinitis. During 1997-1999, more detailed questions about medication use, sleep disturbance, activity limitation and need for urgent medical assistance were included.

\section{Lung function and airway responsiveness}

In 1982 spirometric function was measured using Vitalograph dry-bellows spirometers (Vitalograph Ltd, Buckingham, UK) and tracings read manually. Forced expiratory manoeuvres were repeated until two readings of forced expiratory volume in one second (FEV1) and forced vital capacity (FVC) within $100 \mathrm{~mL}$ were obtained, of which the largest value was used in analyses. Subjects who had taken a $\beta$-agonist within $6 \mathrm{~h}$ of presenting were asked to withhold medication before returning for later testing. Subjects with an FEV1/FVC ratio of $<0.8$ prior to bronchodilator were classified as having obstructive spirometric function.

A histamine inhalation test was administered using the rapid method [15]. In 1982, histamine was administered using De Vilbiss no 40 (Sunrise Medical, Carlsbad, CA, USA) glass hand-held nebulisers in doubling or quadrupling doses starting at $0.03 \mu \mathrm{mol}$. AHR was defined as $\mathrm{a} \geqslant 20 \%$ fall in FEV1 at at cumulative histamine dose of $\leqslant 3.9 \mu \mathrm{mol}$. Subjects who had a baseline FEV1 of $<60 \%$ were not given histamine but instead were given $200 \mu \mathrm{g}$ of salbutamol, and 10 min later lung function was again measured. An increase in FEV1 of $\geqslant 15 \%$ was taken as a positive bronchodilator response [16] and these subjects were also classified as having AHR.

\section{Atopy}

Skin-prick test reactions to common allergens were measured on the forearm [17]. The allergens used in 1982 were: Dermatophagoides farinae and D. pteronyssinus, house dust, cat dander, cockroach, ryegrass, plantain and Alternaria tenuis (Hollister-Stier). Histamine and glycerol were used as positive and negative controls. Weal size was recorded as the long axis and its perpendicular and mean weal size was used in analyses. A mean weal size of $\geqslant 3 \mathrm{~mm}$ was considered a positive test result. Subjects with a positive response to any one or more allergens were defined as atopic.

\section{Definitions of asthma in adult life}

Two clinical definitions of asthma were utilised as outcomes, based on responses to the 1997-1999 questionnaire. Subjects were classified as having "asthma symptoms" if they reported wheeze, sleep disturbance due to asthma or inhaled steroid use in the 12 months prior to study. A subgroup of these subjects were defined as having "troublesome asthma" if they had had either a hospital attendance, an urgent doctor visit, activity limitation or sleep disturbance due to asthma in the 12 months prior to study.

\section{Statistical analyses}

Between-group means were compared using unpaired t-tests and between-group proportions were compared using the Chi-squared test. Multivariable logistic regression was performed with stepwise selection of covariates. Variables were kept in the model if their $\mathrm{p}$-value was $<0.25$. Odds ratios (ORs) are reported with $95 \%$ confidence intervals (CI). For all hypothesis tests, $\mathrm{p}$-values $<0.05$ were regarded as statistically significant.

For both outcomes, i.e. asthma symptoms and troublesome asthma, LRs for the presence of each characteristic were calculated as the ratio of the prevalence of the characteristic among those who had the disease outcome to the prevalence of the characteristic among those who did not have the disease outcome. LRs for the absence of each characteristic were calculated analogously, as using the proportion of subjects without the characteristic among those with and without the disease outcome. Multivariate, or independent, LRs and CIs were calculated by adjusting the original, univariate LRs using shrinkage factors derived from a weighted multivariate logistic regression model $[13,14]$.

\section{Results}

Questionnaire data were available for $80 \%$ of the cohort at age 23-27 yrs. Table 1 shows the childhood characteristics of subjects who returned for follow-up during 1997-1999 compared with subjects who did not return for follow-up. The table shows that there were no significant differences between the groups in terms of age, prevalence of atopy, recent wheeze, AHR or asthma diagnosis. Although a greater proportion of females were tested during the 1997-1999 follow-up, this difference was not statistically significant.

Table 2 shows the unadjusted (univariate) and adjusted

Table 1.-Baseline characteristics of those who attended follow-up as adults compared with those who were unavailable for follow-up

$\begin{array}{cc}\begin{array}{c}\text { Available for } \\ \text { follow-up }\end{array} & \begin{array}{c}\text { Not available } \\ \text { for follow-up }\end{array}\end{array}$

\begin{tabular}{lccl}
\hline Subjects n & 575 & 143 & \\
Females & $49.0(44.9-53.1)$ & $41.3(33.3-49.3)$ & 0.09 \\
Age yrs & $8.5(8.4-8.6)$ & $8.6(8.5-8.7)$ & 0.6 \\
Atopy & $22.7(19.3-26.1)$ & $22.4(15.6-29.2)$ & 0.9 \\
Recent wheeze & $10.4(7.9-12.9)$ & $10.5(5.5-15.5)$ & 0.9 \\
$\begin{array}{l}\text { Airway } \\
\quad \text { hyperresponsiveness }\end{array}$ & $9.8(7.3-12.2)$ & $7.0(2.8-11.2)$ & 0.3 \\
$\begin{array}{l}\text { Diagnosis of asthma } \\
\text { y }\end{array}$ & $9.5(7.1-11.9)$ & $7.8(3.4-12.2)$ & 0.5 \\
\hline
\end{tabular}

Data are presented as percentage $(95 \%$ confidence interval) unless otherwise stated. 
Table 2. - Odds ratios (OR) for having asthma symptoms or troublesome asthma as young adults aged 23-25 yrs if the shown characteristic was present at age 8-10 yrs

\begin{tabular}{|c|c|c|c|c|c|c|c|c|}
\hline \multirow[t]{2}{*}{ Childhood characteristic } & \multicolumn{4}{|c|}{ Asthma Symptoms $(\mathrm{n}=200)$} & \multicolumn{4}{|c|}{ Troublesome Asthma ( $n=82)$} \\
\hline & Unadjusted OR & p-value & Adjusted OR & p-value & Unadjusted OR & p-value & Adjusted OR & p-value \\
\hline Atopy & $3.2(2.1-4.9)$ & $<0.001$ & $2.6(1.6-4.0)$ & $<0.001$ & $4.0(2.5-6.6)$ & $<0.001$ & $4.0(2.3-7.2)$ & 0.0001 \\
\hline AHR & $5.1(2.8-9.4)$ & $<0.001$ & $2.9(1.5-5.6)$ & 0.002 & $3.7(2.0-6.8)$ & $<0.001$ & & \\
\hline Recent wheeze & $4.1(2.3-7.2)$ & $<0.001$ & $2.1(1.1-5.0)$ & 0.02 & $4.0(2.2-7.2)$ & $<0.001$ & & \\
\hline FEV $1 /$ FVC ratio $<80 \%$ & $3.4(1.3-8.6)$ & 0.02 & $3.0(1.0-9.1)$ & 0.05 & $3.7(1.4-9.8)$ & 0.008 & $4.1(1.4-12.3)$ & 0.02 \\
\hline Female & $1.3(0.9-1.8)$ & 0.2 & $1.7(1.1-2.4)$ & 0.008 & $1.9(1.1-3.0)$ & 0.01 & $3.2(1.8-5.7)$ & 0.0001 \\
\hline Diagnosed asthma & $2.6(1.5-4.6)$ & 0.001 & & & $2.1(1.1-4.0)$ & 0.03 & & \\
\hline Eczema & $1.6(1.1-2.4)$ & 0.03 & & & $2.2(1.3-3.6)$ & 0.003 & & \\
\hline Hayfever & $2.6(1.7-3.9)$ & $<0.001$ & & & $4.1(2.5-6.8)$ & $<0.001$ & $2.9(1.7-5.1)$ & 0.0002 \\
\hline Bronchitis before age 2 yrs & $1.9(1.3-2.9)$ & 0.002 & & & $2.4(1.4-3.9)$ & 0.002 & & \\
\hline Maternal asthma & $1.2(0.7-2.1)$ & 0.5 & & & $1.1(0.5-2.4)$ & 0.8 & & \\
\hline Maternal allergies & $1.1(0.8-1.6)$ & 0.5 & & & $1.5(0.9-2.3)$ & 0.1 & & \\
\hline Paternal asthma & $1.6(0.8-2.9)$ & 0.2 & & & $2.4(1.2-4.8)$ & 0.02 & & \\
\hline Paternal allergies & $1.0(0.7-1.4)$ & 0.9 & & & $1.2(0.7-2.0)$ & 0.4 & & \\
\hline
\end{tabular}

Data are presented as OR (95\% confidence interval) unless otherwise stated. AHR: airway hyperresponsiveness; FEV1: forced expiratory volume in one second; FVC: forced vital capacity. Variables were included in the final model if $p<0.05$. The variables in the final model were adjusted for each other.

(multivariate) ORs for childhood characteristics predicting asthma symptoms and troublesome asthma in adulthood. There were 200 subjects $(34.7 \%$ of the cohort) who had asthma symptoms in adulthood. In the asthma symptoms group, 95\% had wheeze, $22 \%$ had used inhaled steroid and $35 \%$ had sleep disturbance at any time in the last 12 months. A total of 82 of the 200 subjects with asthma symptoms ( $14.3 \%$ of the cohort and $41 \%$ of the asthma symptoms group) were classified as having troublesome asthma. In the troublesome asthma group, $84 \%$ had sleep disturbance, $50 \%$ had activity limitation, $11 \%$ had an urgent doctor visit and $7 \%$ had a hospital attendance at any time during the last 12 months. Independent predictors of asthma symptoms in adult life were being female and having atopy, AHR, recent wheeze and abnormal FEV1/FVC ratio at age 8-10 yrs. Independent predictors of troublesome asthma in adult life were being female and having atopy, an abnormal FEV1/FVC ratio and hayfever at age $8-10$ yrs.

Table 3 shows the adjusted (multivariate) LRs for asthma symptoms and troublesome asthma in adulthood for the presence and absence of childhood characteristics that were significant predictors in the multivariate models. The strongest, independent positive predictors of the presence of asthma symptoms in adulthood were the presence of obstructive spirometry and the presence of AHR in childhood. However, only a minority of subjects with these outcomes in adult life had the predictor characteristics present in childhood.
Consequently, the negative LRs were only slightly $<1$, indicating that the absence of any attribute did not substantially reduce the overall likelihood of having asthma in adult life. The strongest positive predictors for troublesome asthma were the presence of obstructive spirometry and atopy in childhood. Being male was the strongest independent predictive factor for the absence of troublesome asthma in adult life.

Cumulative (or overall) multivariate LRs can be calculated by making use of information about the presence or absence of each particular childhood characteristic in an individual. Table 4 depicts a sample of possible combinations for the outcome of asthma symptoms. For example, a young female, who is atopic, has AHR, recent wheeze and obstructive spirometry, has a multivariate LR of 36.9 for having asthma symptoms in adulthood. A young male who reports none of these characteristics is much less likely to develop asthma symptoms as an adult and has an LR of asthma symptoms in adulthood of 0.48 .

\section{Discussion}

This study has demonstrated that it is possible to predict the likelihood of an individual manifesting various features of asthma in adult life, based on characteristics that can be assessed in childhood. It extends previous observations in this

Table 3. - Multivariate likelihood ratios (LR) for childhood characteristics predicting outcomes in adulthood

\begin{tabular}{|c|c|c|c|c|c|c|}
\hline \multirow[t]{2}{*}{ Childhood characteristics } & \multicolumn{3}{|c|}{ Asthma symptoms } & \multicolumn{3}{|c|}{ Troublesome asthma } \\
\hline & $\begin{array}{c}\text { With } \\
\text { characteristic } \%\end{array}$ & LR present & LR absent & $\begin{array}{c}\text { With } \\
\text { characteristic } \%\end{array}$ & LR present & LR absent \\
\hline Atopy & 36.7 & $2.02(1.54-2.66)$ & $0.79(0.67-0.94)$ & 47.6 & $2.56(1.77-3.72)$ & $0.64(0.47-0.88)$ \\
\hline AHR & 19.6 & $2.56(1.78-3.67)$ & $0.89(0.79-1.01)$ & & & \\
\hline Wheeze in last 12 months & 19.5 & $1.92(1.46-2.53)$ & $0.92(0.83-1.02)$ & & & \\
\hline Obstructive spirometry & 5.5 & $2.88(1.27-6.53)$ & $0.96(0.83-1.12)$ & 8.5 & $4.36(1.72-11.04)$ & $0.93(0.73-1.18)$ \\
\hline Female & 53.0 & $1.29(0.78-2.13)$ & $0.77(0.46-1.30)$ & 62.2 & $1.69(0.96-2.89)$ & $0.53(0.27-1.06)$ \\
\hline Hayfever & & & & 45.6 & $2.14(1.59-2.89)$ & $0.72(0.57-0.91)$ \\
\hline
\end{tabular}

Data are presented as LR (95\% confidence interval) unless otherwise stated. For asthma symptoms, there were 200 subjects and for troublesome asthma, $\mathrm{n}=82$. AHR: airway hyperresponsiveness. 
Table 4.-Examples of the estimation of cumulative (overall) multivariate likelihood ratios (LR) for the prediction of "asthma symptoms" in adulthood, based on various combinations of characteristics in childhood

\begin{tabular}{|c|c|c|c|c|c|c|c|c|c|c|}
\hline \multicolumn{2}{|l|}{ Atopy } & \multicolumn{2}{|c|}{ AHR } & \multicolumn{2}{|c|}{ Recent wheeze } & \multicolumn{2}{|c|}{ Obstructive spirometry } & \multicolumn{2}{|c|}{ Female } & \multirow{2}{*}{$\begin{array}{c}\text { Cumulative } \\
\text { multivariate LR }\end{array}$} \\
\hline Present & LR & Present & LR & Present & LR & Present & LR & Present & LR & \\
\hline Yes & 2.02 & Yes & 2.56 & Yes & 1.92 & Yes & 2.88 & Yes & 1.29 & 36.9 \\
\hline Yes & 2.02 & Yes & 2.56 & Yes & 1.92 & Yes & 2.88 & No & 0.77 & 22.0 \\
\hline Yes & 2.02 & Yes & 2.56 & Yes & 1.92 & No & 0.96 & Yes & 1.29 & 12.3 \\
\hline Yes & 2.02 & Yes & 2.56 & No & 0.92 & No & 0.96 & No & 0.77 & 3.5 \\
\hline No & 0.79 & Yes & 2.56 & Yes & 1.92 & No & 0.96 & Yes & 1.29 & 4.8 \\
\hline Yes & 2.02 & No & 0.89 & No & 0.92 & No & 0.96 & No & 0.77 & 1.2 \\
\hline No & 0.79 & No & 0.89 & No & 0.92 & No & 0.96 & Yes & 1.29 & 0.80 \\
\hline No & 0.79 & No & 0.89 & No & 0.92 & No & 0.96 & No & 0.77 & 0.48 \\
\hline
\end{tabular}

AHR: airway hyperresponsiveness.

cohort describing risk factors for progression and remission of atopy, wheeze and AHR during childhood [9]. The multivariate LRs presented here, when used in combination, will be valuable in providing individual prognostic information and also in selecting and stratifying subjects for future trials of preventive interventions.

The follow-up rate of $80 \%$ for this cohort indicates that selection bias at the final follow-up study was minimised. The majority of the $20 \%$ of subjects who were not available at follow-up were uncontactable and there was no reason to believe that this group differed significantly from those whom the authors could contact. Furthermore, there was no statistically significant difference in the baseline characteristics of those who attended or were lost to follow-up.

The childhood characteristics identified as being significant predictors of asthma in adulthood in this study were consistent with the reports of other longitudinal studies. For example, in the Tasmanian cohort study, being female, having parents with asthma, and having eczema and/or airflow obstruction at age $7 \mathrm{yrs}$, were significant independent predictors of asthma at 29-32 yrs of age [10]. In the Dutch longitudinal study of 119 asthmatic children, low lung function and the presence of AHR were both significant predictors of current asthma symptoms in adulthood [7]. Hence, there are external data to support the validity of the multivariate model on which the LRs are based. There are few comparable published data on predictors of troublesome asthma. Among a New Zealand cohort of subjects who had wheezed at any time, atopy, low lung function and AHR were all predictors of hospital admission and, in contrast to the present findings, being male was also a predictor of this adverse outcome [11].

The present study found that the presence and absence of predictive characteristics were not symmetrical in their impact on risk of asthma outcomes. Although the presence of atopy, recent wheeze, abnormal FEV1/FVC ratio and AHR substantially increased the odds of having asthma in adulthood, their absence had a much smaller impact in reducing the odds. Most adults who had asthma symptoms in 1997-1999 had neither atopy, recent wheeze, AHR nor abnormal lung function during childhood. This is consistent with the previous observation in that cohort indicating that propensity to develop asthma is not necessarily expressed by the time a child is 8-11 yrs of age [9].

The effect of treatment on asthma outcomes has not been addressed in this paper. Very few of the children were using inhaled steroids during 1982. Also, of the 200 participants who were classified as having asthma symptoms in adulthood, only $44(22 \%)$ had used inhaled steroids in the previous 12 months. Of the 82 participants who were classified as having troublesome asthma, only $34(42 \%)$ had used inhaled steroids in the previous 12 months. This suggests that asthma was undermanaged in this cohort and may help to explain the high proportion of participants who were classified as having troublesome asthma. More appropriate treatment of childhood asthma may help to reduce the burden of asthma in adulthood [10, 18]. In the future, the study of prospective cohorts will assist with the assessment of whether more aggressive management leads to a reduction in the incidence and/or severity of asthma.

A novel feature of this study is the estimation of multivariate LRs. The fact that the LRs have been adjusted for each other means that they are independent of each other and can be multiplied together to give an overall LR [13] for an individual. JAESCHKE et al. [19] have published a useful article to describe the interpretation of an LR in a clinical setting [19]. For any individual, the overall LR is calculated by multiplying together the LRs corresponding to the combination of characteristics observed in that individual. Examples for the outcome asthma symptoms are shown in table 4. This overall individual LR represents the extent to which the prior odds of the outcome are increased (if $L R>1$ ) or decreased (if $\mathrm{LR}<1$ ) by information about the risk factors. In this context, and in the absence of any additional information about the individual's risk status, the prior odds can be regarded as a simple transformation of the population prevalence of the outcome, and hence the probability of an individual having the outcome can be deduced. Confirmation of the validity of this prediction model presented here will require replication in another, independent population.

To conclude, the authors found that in individuals with wheeze during childhood, those mostly likely to have problems with asthma in adult life, and hence with most to gain from preventive interventions, were those who had obstructive spirometry, airway hyperresponsiveness and atopy. This confirms the central role of these characteristics in the underlying mechanisms of asthma and the algorithm described here may be used to quantitatively predict the prognosis for asthma outcomes in individual children.

\footnotetext{
Acknowledgements. The authors acknowledge the important contribution of the late A.J. Woolcock to the work presented here. They would also like to thank all the project coordinators and all the research assistants who helped to follow-up and test this cohort. Finally, the authors thank the members of the cohort who have allowed them to interupt their lives every 2 yrs to collect these valuable data.
} 


\section{References}

1. Williams H, McNicol KN. Prevalence, natural history, and relationship of wheezy bronchitis and asthma in children. An epidemiological study. BMJ 1969; 4: 321-325.

2. Gibson HB, Silverstone H, Gandevia B, Hall GJL. Respiratory disorders in seven-year-old children in Tasmania. Aims, methods and administration of the survey. Med J Aust 1969; 2: 201-205.

3. Peat JK, Salome CM, Sedgwick CS, Kerrebijn J, Woolcock AJ. A prospective study of bronchial hyperresponsiveness and respiratory symptoms in a population of Australian schoolchildren. Clin Exp Allergy 1989; 19: 299-306.

4. Sears MR, Jones DT, Silva PA, Simpson A, Williams SM. Asthma in seven year old children: a report from the Dunedin Multidisciplinary Child Development Study. NZ Med J 1982; 95: 533-536.

5. Lewis S, Butland B, Strachan D, et al. Study of the aetiology of wheezing illness at age 16 in two national British birth cohorts. Thorax 1996; 51: 670-676.

6. Bodner C, Ross S, Douglas G, et al. The prevalence of adult onset wheeze: longitudinal study. BMJ 1997; 314: 792-793.

7. Gerritsen J, Koeter GH, Postma DS, Schouten JP, Knol K. Prognosis of asthma from childhood to adulthood. Am Rev Respir Dis 1989; 140: 1325-1330.

8. Phelan PD, Robertson CF, Olinsky A. The Melbourne Asthma Study: 1964-1999. J Allergy Clin Immunol 2002; 109: 189-194.

9. Xuan W, Marks GB, Toelle BG, et al. Risk factors for onset and remission of atopy, wheeze, and airway hyperresponsiveness. Thorax 2002; 57: 104-109.

10. Jenkins MA, Hopper JL, Bowes G, Carlin JB, Flander LB,
Giles GG. Factors in childhood as predictors of asthma in adult life. BMJ 1994; 309: 90-93.

11. Rasmussen F, Taylor DR, Flannery EM, et al. Risk factors for hospital admission for asthma from childhood to young adulthood: a longitudinal population study. J Allergy Clin Immunol 2002; 110: 220-227.

12. Sackett DL, Haynes R, Guyatt G, Tugwell P. Clinical Epidemiology: a Basic Science for Clinical Medicine. 2nd Edn. Boston, Little Brown and Company, 1991

13. Spiegelhalter DJ, Knill-Jones RP. Statistical and knowledgebased approaches to clinical decision-support systems, with an application in gastroenterology. J R Statist Soc A 1984; 147: 35-77.

14. Straus SE, McAlister FA, Sackett DL, Deeks JJ. The accuracy of patient history, wheezing, and laryngeal measurements in diagnosing obstructive airway disease. JAMA 2000 ; 283: $1853-1857$.

15. Yan K, Salome C, Woolcock AJ. Rapid method for measurement of bronchial responsiveness. Thorax 1983; 38: 760-765.

16. American Thoracic Society. Lung function testing: selection of reference values and interpretative strategies. Am Rev Respir Dis 1991; 144: 1202-1218.

17. Pepys J. Skin tests in diagnosis. In: Gell PGH, Coombs RRA, Lachman PJ, eds. Clinical Aspects of Immunology. 3rd Edn. Oxford, Blackwell Scientific Publications, 1975; pp. $55-80$.

18. Roorda RJ, Gerritsen J, van Aalderen WMC, et al. Risk factors for the persistence of respiratory symptoms in childhood asthma. Am Rev Respir Dis 1993; 148: 1490-1495.

19. Jaeschke R, Guyatt G, Sackett DL. Users' guides to the medical literature: III. How to use an article about a diagnostic test: B. What are the results and will they help me in caring for my patients? JAMA 1994; 271: 703-707. 